\title{
Level set based Immersed Boundary method and its application to CFD simulation for moving boundary problem
}

\author{
Deepak. S. Patil ${ }^{1 *}$, Atul Sharma ${ }^{2}$, Namshad $\mathrm{T}^{2}$ and Tapobrata Dey ${ }^{1}$ \\ ${ }^{1}$ Dept. of Mechanical Engineering, D Y Patil College of Engineering, Akurdi, SPPU, Pune 44, India \\ ${ }^{2}$ Dept. Of Mechanical Engineering, Indian Institute of Technology Bombay, Mumbai-76, India \\ Accepted 15 June 2016, Available online 20 June 2016, Special Issue-5 (June 2016)
}

\begin{abstract}
A novel level set Immersed Boundary method is used for transient Computational Fluid Dynamics simulation of flow across a moving/rigid Immersed boundary. At various time instants level-set is used to capture moving boundaries accurately with proper numerical implementation of boundary conditions and conservation laws in fluid regions. An in-house code is used to study four different problems ranging from a flow over a stationary circular cylinder, a rotating circular cylinder, pitching of airfoil and an undulating swimming fish-like undulating body with amplitude variations.
\end{abstract}

Keywords: Immersed Boundary method, Level-set method, Stationary circular cylinder, rotating cylinder, AirfoilNACA0012, Thrust coefficient, Strouhal number, Computational Fluid Dynamics (CFD), vortices.

\section{Introduction}

In present day of scientific studies and vast industrialization need for underwater exploration is in increasing demand. Its need is felt in vast areas like oil extraction, fisheries acoustics, platonic movement and many others. Still the physics involving the undulation of fishes needs to be studied thoroughly to understand that how fishes take advantage of formation of vortex behind their body. To do so, numerical techniques are developed which should be less expensive and should be having flexibility in using governing equations as is not the case with experiments.

Computational Fluid Dynamics has proved to be an effective tool that allows investigators to perform simulation and collect data that may only be possible by traditional physical experiments. The simulation includes many variations in parameters as the flow progresses and its effects on Reynolds number, amplitude, pitching angles, phase difference, frequency, Strouhal number and their effects on drag, thrust, and lift coefficients and also on propulsive efficiency.

In earlier days for the simulation of complex bodies Cartesian grid with finite difference method was used which approximated the curved body as stepped one. In this simulation curved surfaces were approximated either by horizontal or vertical lines. So, for better accuracy there was a need to modify the method and a new method of body-fitted curvilinear structured grid with finite-volume method came to existence and became more popular than finite-difference.
In case of body-fitted structured and unstructured grid, the control volume generated is either in solid or fluid; with solid-fluid interface coinciding with the common face of border control volume i.e. at least one neighbor grid point is in other phase. At the interface the implementation of boundary conditions is easy as it is simpler to each of the grid points in solid and fluid control volumes. Whereas in the case of non-body fitted Cartesian grid (Shrivastava), certain control volume filled with both solid and fluids are found, so a special treatment is required to deal with such cases.

To model complex problems involving moving body flow, remeshing at each time step is required which is not the case with fixed grid problems, but the computational time required for grid generation in fixed grid is less as compared to moving grid and bodyfitted Cartesian grid; as there is a change in the position of grid points as time progress. So, to account for all such problems, an Immersed boundary method was introduced by (Peskin) to study the blood flow in human heart.

Level-set method (Sussman) is a powerful numerical technique for analyzing and computing moving boundaries in variety of situations. It relies on Eulerian rather than lagrangian method of tracking interface and thus is easy to implement numerically. Choi (2007), Tan (2010), Yang (2009), Cirak (2007), Cottet (2006), Shrivastava (2013), Legay (2006) have used level set method with a discrete forcing function. The present work uses a sharp interface level set method as diffused interfaces causes smoothening of discontinuities at the interface resulting in parasitic 
flows Udaykumar (2001) and the forcing function is also eliminated, thus making present numerical simulation more physics based and simpler to implement numerically. The present method is used to capture transient movement of solid-fluid interface (boundary of the solid) using level-set method and transient flow of fluid using Navier-Stoke solver, in a fixed Cartesian non-body fitted grid, with direct implementation of boundary conditions in the finite volume method. The scope of the present work is to validate a Level set method based IB method using CFD code for the cylinder and NACA0012 shapes. It also investigates the physical significance of formation of vortices and its effects on thrust generation. The study follows with the variation of amplitude of fish like motion on thrust generation, along with the variation of Strouhal number and finally investigating different parameters which influence the thrust generation of the structure.

\section{Numerical Simulation}

Figure 1 shows the computational domain considered for the study. The body of the fish is considered as a NACA0012 airfoil. The translating motion for the fish is modeled by giving an inlet flow velocity equal to the transverse velocity of the body. The figure depicts the undulating fish-like locomotion with no slip boundary conditions and a uniform free stream velocity, with a domain height of 8c, upstream length $5 \mathrm{c}$ and downstream length 11c.

A two dimensional simulation is carried out using level-set immersed boundary method in which the following governing equations are solved.

\section{Fluid:}

Continuity:

Momentum:

$$
\nabla \cdot \vec{U}=0
$$

$$
\frac{\partial \vec{U}}{\partial \tau}+\nabla \cdot(\vec{U} \vec{U})=-\nabla P+\frac{1}{R e} \nabla^{2} \vec{U}
$$

Solid:

Level set:

$$
\frac{\partial \Phi}{\partial \tau}+\vec{U}_{a d v} \cdot \nabla \Phi=0
$$

Re-initialization:

$$
\frac{\partial \Phi}{\partial \tau_{s}}+S_{€}\left(\Phi_{0}\right)\left(\left|\nabla \Phi_{0}\right|-1\right)=0
$$

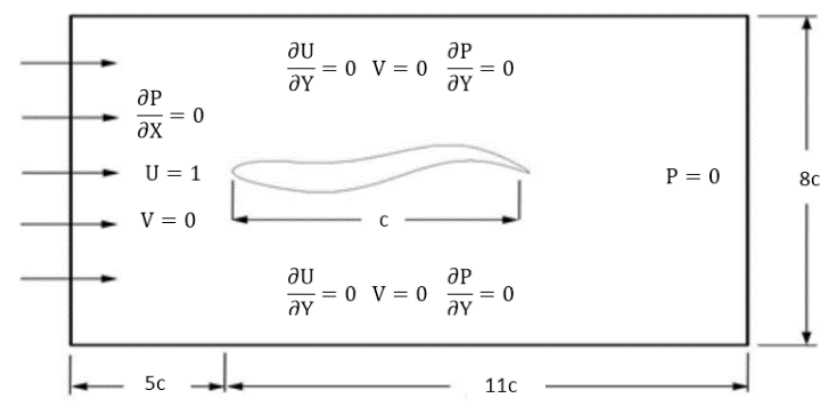

Fig.1 Computational domain and boundary conditions of NACA0012 hydrofoil
Where $\vec{U}_{a d v}$ is advecting velocity, $\tau_{s}$ is pseudo time and $S_{€}\left(\Phi_{0}\right)$ is smoothened sign function.

Continuity and momentum equations are solved in the fluid domain to obtain the velocity and pressure distribution in the fluid domain whereas the interface movement is traced using Level set and re-initialization equations (Shrivastava). In this method, a normal distance function called level set function $(\Phi)$ is used to distinguish between solid zone $(\Phi<0)$ and fluid zone $(\Phi>0)$. Figure 2 shows the same. The grid points in fluid and solid CV's are showed with unfilled circles and squares respectively, while filled symbol for the grid points of border CV's and triangular symbols represents face center in between fluid and solid border $\mathrm{CV}$.

The non-dimensional parameters used are Reynolds number, Strouhal number, and nondimensional maximum amplitude at the tail. The Reynolds number is defined as:

$$
R e=\frac{U c}{v}
$$

Where $\mathrm{U}$ is the free-stream flow velocity; $\mathrm{c}$ is the chord length of the airfoil and $v$ is the kinematic viscosity. Strouhal number is defined based on the maximum amplitude at the tail and the undulating frequency.

$$
S t=\frac{f a_{\max }}{U_{\infty}}
$$

Where $a_{\max }$ is maximum undulating amplitude of tail and $f$ is the frequency of undulation. Vortex shedding causes two forces viz. drag force and lift force acting on surface of the hydrofoil Lift; $\mathrm{F}_{\mathrm{L}}$, is the force component in the stream-normal direction, while drag; $F_{D}$, is the force component in the stream-wise direction. These forces can be expressed non-dimensionally by the lift coefficient $\left(\mathrm{C}_{\mathrm{L}}\right)$, and drag coefficient $\left(\mathrm{C}_{\mathrm{D}}\right)$, respectively:

$$
\begin{aligned}
C_{L} & =\frac{2 F_{L}}{\rho U^{2} c} \\
C_{D} & =\frac{2 F_{D}}{\rho U^{2} c}
\end{aligned}
$$

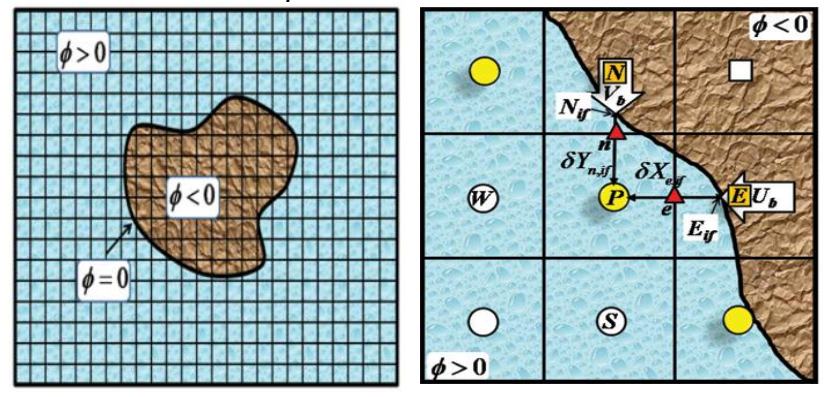

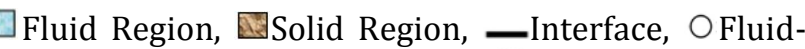
grid point, $\square$ Solid grid point, OFluid-Border grid point, $\square$ Solid-Border grid point, $\Delta$ Border-face grid point

Fig.2 Grid generation and different types of Control volumes (Shrivastava)

Fig 3 shows a non-uniform Cartesian grid of $768 \times 314$ nodes is used for simulation. The grid consist of outer 
domain of coarse grid and inner domain of fine grid with a time step of 0.001 .

\section{Validation Study}

To study and test an in-house code based on level-set method on various moving boundary problems, a validation study is being performed on cylinder (stationary and rotating) and NACA0012. A computational grid, fig 4, signifies that grid is made more fine near the body as compared to outer domain.

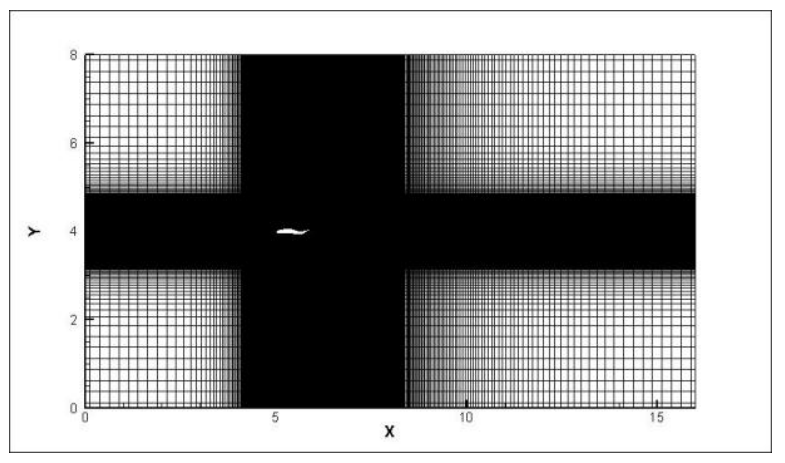

Fig.3 Grid generation domain of NACA0012
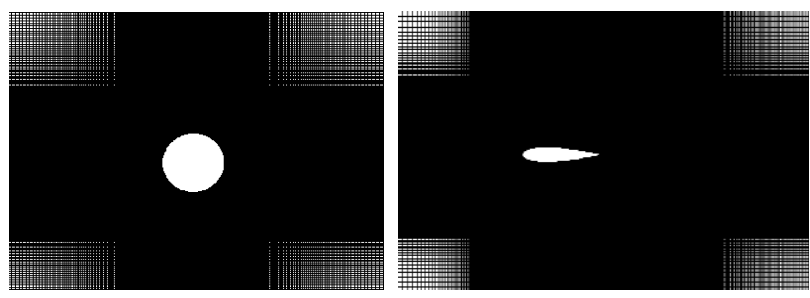

Fig.4 Grid generation near the cylinder and an airfoil (NACA0012)

\subsection{CFD simulation of Flow over a Circular Stationary Cylinder.}

In this problem a circular cylinder is embedded in a finite size domain with a grid size of $410 \times 386$ and the flow across the cylinder is assumed to be uniform. A numerical simulation is done for three Reynolds number $(40,100 \& 1000)$ to find the drag and lift coefficient values. A comparison with Calhoun \& Wang work with present work is done for drag and lift coefficient.

Table 1 shows a comparison of drag coefficient values with literature at a Reynolds number of 40 and it can be observed form table that the values of drag and lift coefficient shows a good agreement with the published results and present work, also when value of Reynolds number goes on increasing the value of drag coefficient decreases.

Table1 Drag Coefficient for a $\mathrm{Re}=40$

\begin{tabular}{|c|c|}
\hline Tritton & 1.48 \\
\hline Fornberg & 1.50 \\
\hline Dennis & 1.52 \\
\hline Calhoun & 1.62 \\
\hline Present & 1.62 \\
\hline
\end{tabular}

Fig 5 shows streamlines and vorticity comparison with published and present paper for Reynolds number of 40 and validated that the formation of streamlines and vorticity pattern visualizes that the two streamlines and vortices are perfectly aligned along the mid line of cylinder indicating a stable flow, which is not the case as we increase the value of Reynolds number.
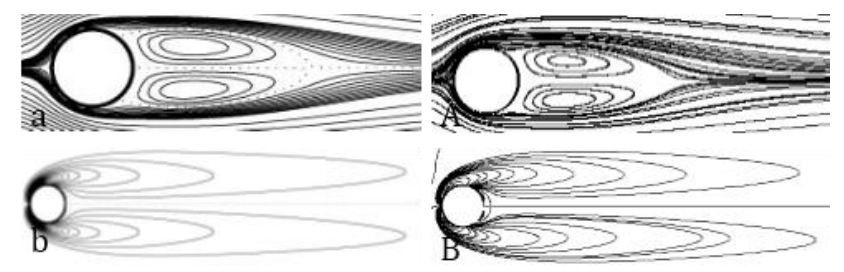

Fig.5 Streamlines and Vorticity of Calhoun and present work at $\mathrm{Re}=40$.

Table 2 shows the marginal range of drag and lift coefficient for Reynolds number of 100 and we can observed a good agreement with the work done by different authors.

Table 2 Drag and Lift Coefficient values at $\mathrm{Re}=100$

\begin{tabular}{|c|c|c|}
\hline & $\mathrm{C}_{\mathrm{D}}$ & $\mathrm{C}_{\mathrm{L}}$ \\
\hline Calhoun & $1.330 \pm 0.014$ & \pm 0.298 \\
\hline Braza & $1.364 \pm 0.015$ & \pm 0.250 \\
\hline Liu & $1.350 \pm 0.012$ & \pm 0.339 \\
\hline Present & $1.395 \pm 0.0011$ & \pm 0.301 \\
\hline
\end{tabular}

As the Reynolds number is increased from 100 to 1000 the value of drag coefficient decreases as can be seen from table 3 , which shows a good result with the values of work done in literature.

Table 3 Drag and Lift Coefficient values at $\mathrm{Re}=1000$

\begin{tabular}{|c|c|c|}
\hline & $\mathrm{C}_{\mathrm{D}}$ & $\mathrm{C}_{\mathrm{L}}$ \\
\hline Present & 1.15 & 1.4 \\
\hline Mittal & 1.46 & 1.35 \\
\hline Roshko & 1.2 & - \\
\hline Wang & 1.14 & 0.54 \\
\hline
\end{tabular}

\subsection{CFD simulation of Flow over a circular rotating Cylinder.}

A validation study is done for a rotating circular cylinder and is numerically validated with literature for a Reynolds number of 100 . In both of the following cases a nondimensional tangential surface velocity ' $\alpha$ ' is varied from 0.4 to 2.5 for a time step of 0.0001 (seen suitable results).

Table 4 shows the value of tangential surface velocity ranging from 0.5 to 2 with change in drag coefficient values and a time step of 0.0001 . Present and Arakeri et.al work shows drag coefficient values similarity with some percentage error and also we can observe that as drag is reduced a thrust (negative value 
of drag coefficient) generation is seen for higher values of $\alpha$.

Table 4 Comparison of present results with Arakeri et.al (2013)

\begin{tabular}{|c|c|c|c|c|}
\hline$\alpha$ & $\Delta \mathrm{t}$ & $\mathrm{C}_{\mathrm{D}}$ & (Present work) $\mathrm{C}_{\mathrm{D}}$ & \% error \\
\hline 0.5 & 0.0001 & 0.79 & 0.87 & 9.19 \\
\hline 1 & 0.0001 & 0.45 & 0.48 & 6.25 \\
\hline 2 & 0.0001 & -0.34 & -0.30 & 11.76 \\
\hline
\end{tabular}

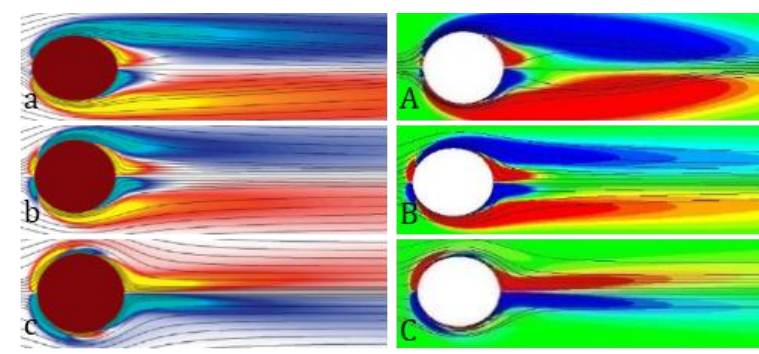

Fig.6 Arakeri and present Streamlines and vorticity isocontours for flow past circular cylinder at $\alpha$ equal to a) 0.5 b) 1 c) 2 and $R e=100$.

Fig. 6 and 7 illustrates the flow field of streamlines and vorticity contours past a circular cylinder. A vonKarman Vortex street is formed in the wake behind of circular cylinder when value of $\alpha$ is low. But as strength of tangential surface velocity increases it leads to a decrease in magnitude of vorticity generated and so vortex sheds in the wake.

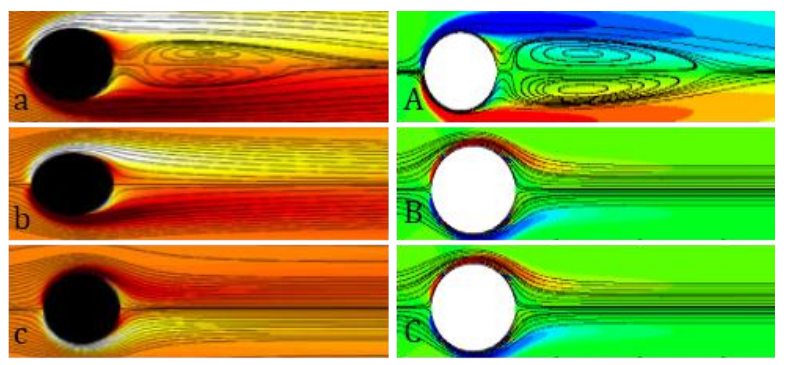

Fig.7 Shukla and present Streamlines and vorticity isocontours for flow past circular cylinder at $\alpha$ equal to a) 0.4 b) 1 c) 2.5 and $R e=100$.

Table 5 shows a precision results for drag coefficient values for present and literature work for a time step of 0.0001 with a percentage error less than $3 \%$ and from this we can observe that as value of $\alpha$ increases to 2.5 , a sign inversion for drag coefficient values is seen, giving a thrust to the body.

Table 5 Comparison of present results with Shukla et.al (2013)

\begin{tabular}{|c|c|c|c|c|}
\hline$\alpha$ & $\Delta \mathrm{t}$ & $\mathrm{C}_{\mathrm{D}}$ & (Present work) $\mathrm{C}_{\mathrm{D}}$ & \% error \\
\hline 0.4 & 0.0001 & 0.889 & 1.001 & 11.11 \\
\hline 1 & 0.0001 & 0.568 & 0.585 & 2.905 \\
\hline 2.5 & 0.0001 & -0.303 & -0.282 & 6.666 \\
\hline
\end{tabular}

\subsection{CFD simulation of flow over a pitching airfoil} (NACA0012).

Table 6 shows a comparison of drag coefficient value with Pedro's paper over one periodic oscillation of an airfoil. It is seen in good agreement with the published work for a Reynolds number of 1100, maximum angular displacement of $5^{\circ}$ and a frequency of undulation of 2.5464. A negative value of drag coefficient points towards thrust generation.

Table 6 Comparison of Pedro and present drag at $\mathrm{Re}=1,100$, maximum angular displacement $\operatorname{Amax}=5^{\circ}$, and frequency 2.5464

\begin{tabular}{|c|c|}
\hline $\mathrm{C}_{\mathrm{D}}$ (Pedro) & $\mathrm{C}_{\mathrm{D}}$ (Present) \\
\hline-0.0964 & -0.08267 \\
\hline
\end{tabular}

Fig 8 illustrates vorticity contours for reynolds number of 1100, maximum angular displacement of $5^{\circ}$ and frequency of 2.5464 , in which blue colour indicates negative votices (fluid rotation in clockwise direection) while red denots positive vortices (fluid rotating in anticlockwise direction). A reverse Von karman Vortex Street can be seen forming indicating a jet flow, responsible for thrust generation.

\section{Results and discussions}

To study variation of flow pattern downstream of hydrofoil a numerical simulation is done for Reynolds number of 4000 and a wavelength of 0.64 while the amplitude is varied from 0.05 to 0.2 and its effects on Strouhal number and thrust coefficient discussed. For simulation purpose in this paper only lowest and a highest values of amplitudes are discussed viz. 0.05 and 0.2. Further graphs are plotted for various values of amplitude and Strouhal number with thrust coefficient.

Fig 9 shows the vorticity contours for $\mathrm{Re}=4000$, $A \max =0.05, \mathrm{St}=0.4$ and $\lambda=0.64$. The variation in the flow pattern downstream of the hydrofoil is shown for one periodic undulation of the tail, with variation of thrust coefficient. It shows downwards motion vorticity contours as seen from figure a1 to a3. Figure a3 shows that vortex is shed when it is on the verge of changing direction of undulation of the tail. Also we can observe that, center of positive vortex shed from bottom surface is above the center of negative vortex shed from the top surface. This is called as reverse vonKarman Vortex Street. The U-velocity in fig 9 indicate a velocity excess indicating stream-wise momentum excess. These both reverse Von-Karman Vortex Street and momentum excess are signs of thrust generation. 


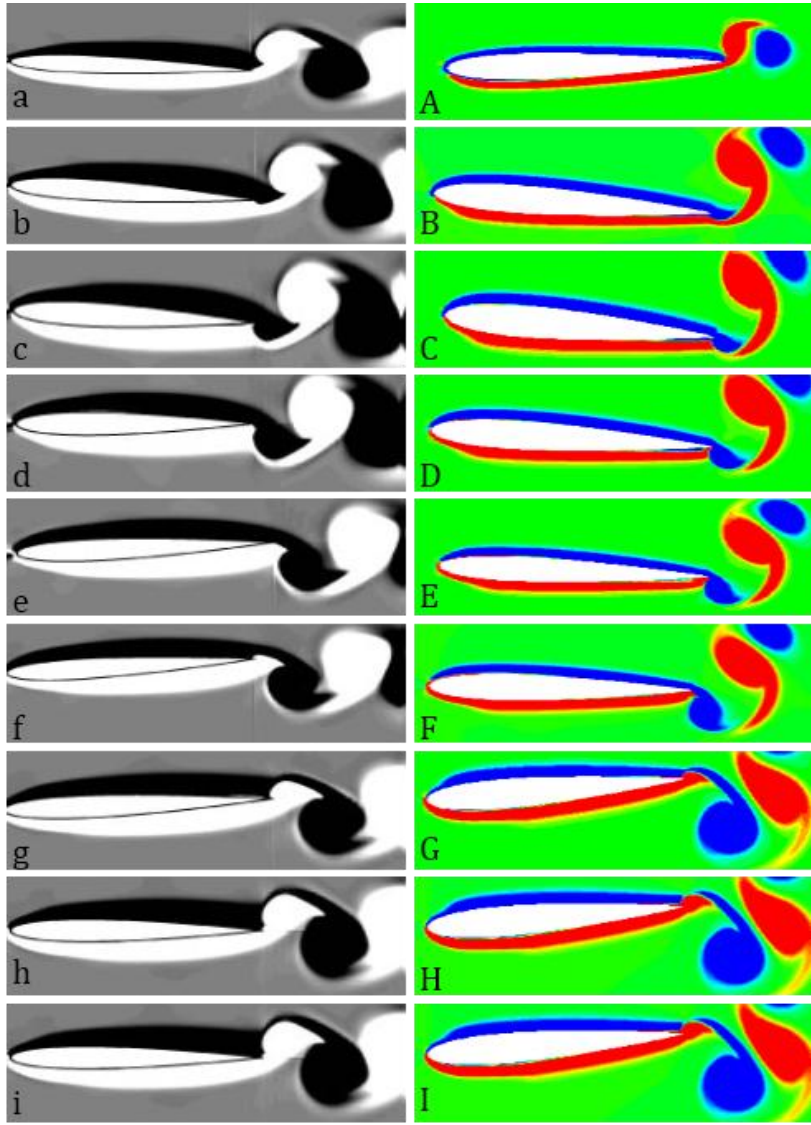

Fig.8 Vorticity contours comparison of Pedro with present work over one cycle with $\mathrm{Re}=1100$ and maximum pitching angle of $5^{\circ}$ and frequency of 2.5464 .

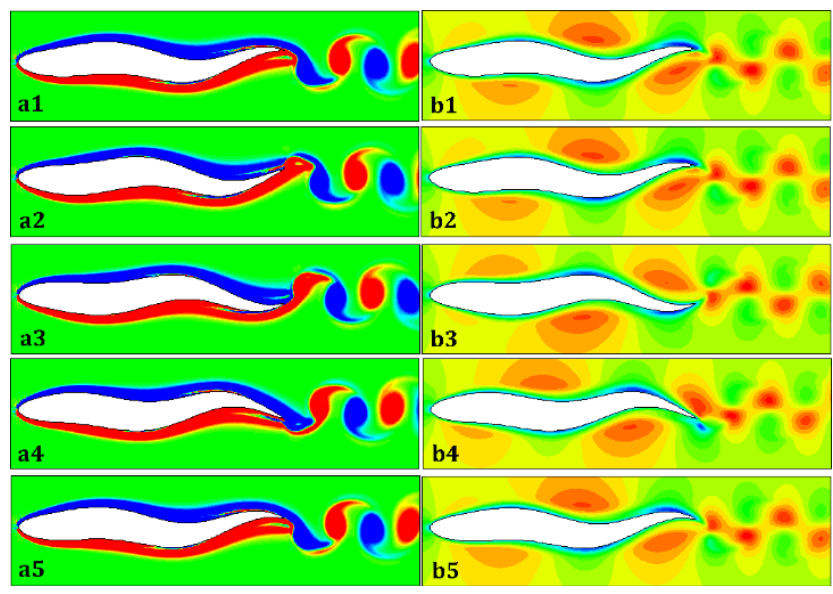

Fig.9 Variations of instantaneous vorticity and Uvelocity contours within one time period of undulation at $\mathrm{Re}=4000, \mathrm{Amax}=0.05, \mathrm{St}=0.4$ and $\lambda=0.64$.

Fig 10 shows a plot of thrust coefficient with time for different position of undulating NACA0012. We can observe five different positions of the body indicating maximum and minimum thrust generation position of the body. Point a2 and a4 shows a very low value of thrust coefficient while point a1, a3 and a5 shows maximum thrust generation which signifies that the vortex is shed.

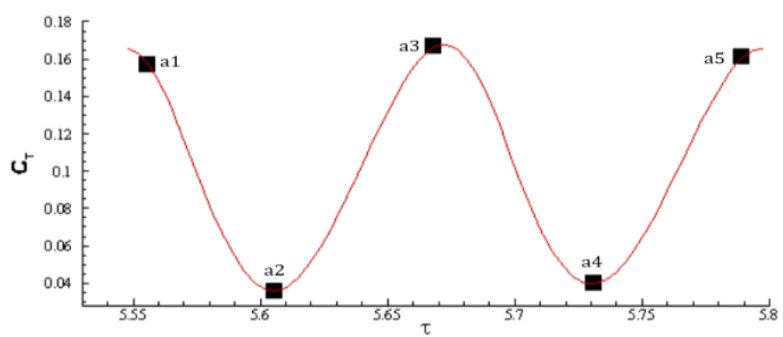

Fig.10 Variation of thrust coefficient with time instantaneous plot

Fig 11 shows the vorticity contours for $\mathrm{Re}=4000$, $A \max =0.2, \mathrm{St}=0.4$ and $\lambda=0.64$. The formation of vortex in the downstream region of hydrofoil signifies that the center of vortex shed from top surface of the hydrofoil lies above the center of the vortex shed from bottom surface. This tells us that Von Karman Vortex is formed behind the hydrofoil and hence drag is generated with a velocity deficient region being formed in the downstream region.

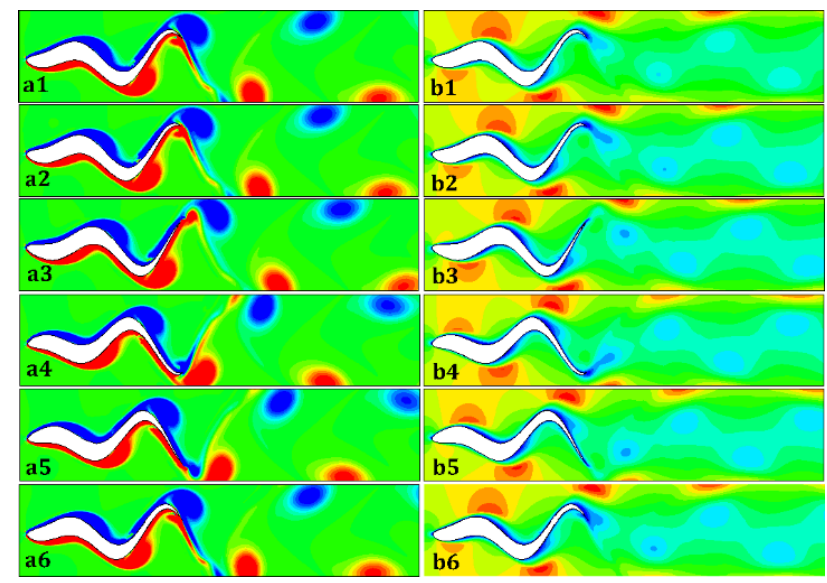

Fig.11 Variations of instantaneous vorticity and Uvelocity contours within one time period of undulation at $\mathrm{Re}=4000, \mathrm{Amax}=0.2, \mathrm{St}=0.4$ and $\lambda=0.64$.

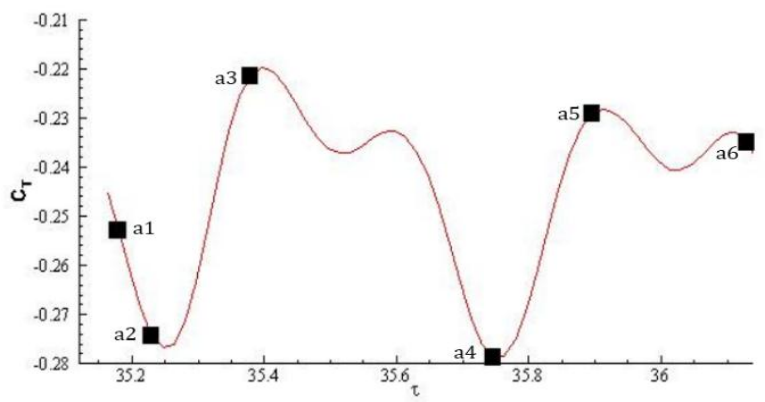

Fig.12 Variation of thrust coefficient with time instantaneous plot

Fig 12 shows a plot of thrust coefficient for one periodic cycle of undulation. From this graph it can be observed that at point a3, a5 and a6, a vortex is shed and we get a maximum value of thrust, while other points (a1, a2, a4) indicates a minimum value of thrust coefficient. 
From fig. 10 and 12 we can conclude that as the amplitude increases from 0.05 to 0.2 , for a $\mathrm{St}=0.4$, $\mathrm{Re}=4000$ and $\lambda=0.64$, the value of thrust coefficient decreases. This can be seen from the vortex patterns generated downstream of the flow, of fig. 9 and 11, as amplitude increases the distance between the centers of the vortex increases and hence the vortices move away from each other. Also the center of positive vortices generated from the bottom surface lies beneath the center of the negative vortices generated from the top surface which is the sign of decrease in amount of thrust generation.

Fig 13 shows a plot of variation of thrust coefficient with change in maximum amplitude of undulation for a Reynolds number of 4000 and wavelength of 0.64 . An increase in thrust coefficient value is with decrease in amplitude (an increase in frequency) and an increase in Strouhal number can be seen from the plot. It is also seen that for lower value of Strouhal (0.2) there is no thrust generation.

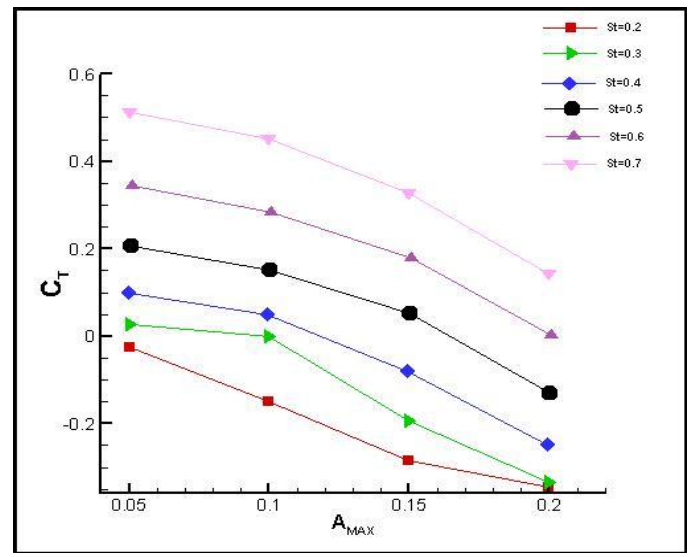

Fig.13 Variation of thrust coefficient with maximum amplitude at $\mathrm{Re}=4000$ and $\lambda=0.64$

So for St=0.2 there is only drag acting on the hydrofoil and we have negative value of thrust coefficient, but as we increase Strouhal number it goes in positive region i.e. a thrust generating hydrofoil.

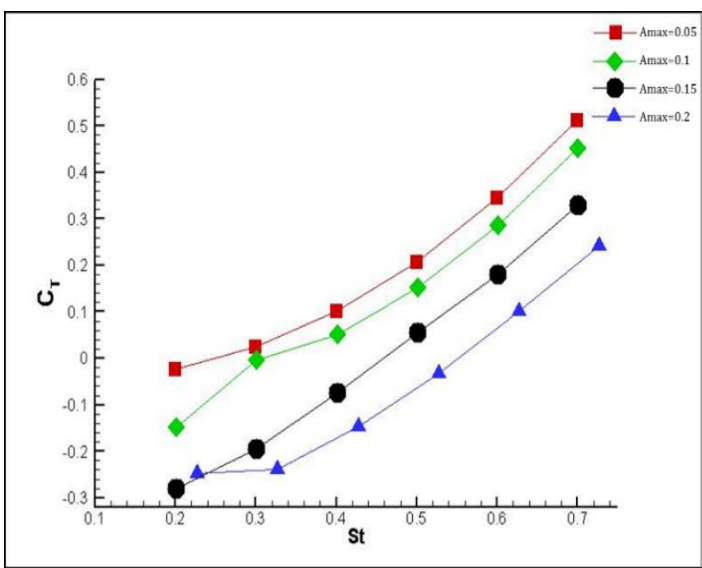

Fig.14 Variation of thrust coefficient with Strouhal number at $\operatorname{Re}=4000$ and $\lambda=0.64$
Fig 14 shows a plot of variation of thrust coefficient with Strouhal number for $\operatorname{Re}=4000$ and $\lambda=0.64$. For lower amplitude we get a maximum thrust generation value, but as amplitude value increases the amount of thrust generation decreases with a decrease in Strouhal number.

\section{Conclusions}

A level-set Immersed Boundary method is employed for simulating the flow over different body shapes tending towards a more streamlined shaped body with an increase in thrust generation. A detailed CFD simulation is undertaken to study effect of amplitude, Reynolds number, Strouhal number and frequency on thrust coefficient. It is observed that reverse Von Karman formed behind the body allows the body to harness energy from the vortices, decreasing their energy expenditure and increasing their efficiency. From fish like locomotion we can say that as amplitude is increased, with a decrease in frequency of undulation, at a certain Strouhal number, the thrust coefficient value decreases. A transition from drag to thrust can be observed from the change in motion of undulation and the shed of vortex.

\section{Acknowledgement}

I would like to thanks all the members of Computational fluid Dynamics and Heat transfer Lab (CFDHT) Dept. of Mechanical Engineering, Indian Institute of Technology, Bombay for their regular support.

\section{References}

I Borazjani, L Ge \& F Sotiropoulos (2008) Curvilinear immersed boundary method for simulating fluid structure interaction with complex 3D rigid bodies, Journal of Computational physics, 227(16), pp.7587-7620.

Mukul Shrivastava, Amit Agrawal \& Atul Sharma (2013) A Novel Level Set-Based Immersed-Boundary Method for CFD Simulation of Moving-Boundary Problems, International Journal of Computation and Methodology, 63 (4), pp.304-326.

Charles S. Peskin, (2002) The Immersed Boundary method, Cambridge University press, Acta Numerica, 11, pp.479-517.

M Sussman, P Smereka \& S Osher, (1994) A level set approach for computing solutions to incompressible two-phase flow Journal of Computational physics, 114(1), pp.146-159.

C. Norberg, (2003) Fluctuating lift on a circular cylinder: review and new measurements, Journal of Fluids and Structures, 17, pp.57-96.

Donna Calhoun, (2002) A Cartesian Grid Method for Solving the Two-Dimensional Stream function-Vorticity Equations in Irregular Regions, Journal of Computational Physics, 176, pp.231-275.

Wang Jia-song, (2010) Flow around a circular cylinder using a Finite-Volume TVD Scheme based on a Vector Transformation Approach, Journal of Hydrodynamics, 22 (2), pp.221-228.

Jaywant H. Arakeri \& Ratnesh K. Shukla, (2013) A unified view of energetic efficiency in active drag reduction, thrust generation and self-propulsion through a loss coefficient with some applications, Journal of Fluids and Structures, 41, pp.22-32.

Ratnesh K. Shukla \& Jaywant H. Arakeri, (2013) Minimum power consumption for drag reduction on a circular cylinder by tangential surface motion, J. Fluid Mech., 715, pp.597-641.

G. Pedro, A. Suleman \& N. Djilali, (2003) A numerical study of the propulsive efficiency of a flapping hydrofoil, International Journal for Numerical Methods in Fluids, 42, pp.493-526. 